\title{
Editoria
}

\section{Die Überprüfung des Erreichens mathematischer Grundkompeten- zen in der Schweiz am Ende der obligatorischen Schulbildung}

\author{
Christian Brühwiler, Pädagogische Hochschule St.Gallen \\ Andrea B. Erzinger, Universität Bern \\ Christian Nidegger, Service de la recherche en éducation (SRED), Genève \\ Miriam Salvisberg, Scuola universitaria professionale della Svizzera italiana
}

Seit rund 25 Jahren wird die Leistungsfähigkeit von Bildungssystemen verstärkt an den Leistungen der Schülerinnen und Schüler gemessen. Diese Wende von der Input- hin zur Outputorientierung geht mit einer stärker evidenzbasierten Rechenschaftslegung von Bildungssystemen einher (Ryan \& Shepard, 2008). Infolgedessen wurden in den letzten Jahrzehnten verschiedene Schulleistungsstudien international unter Beteiligung einer grossen Anzahl von Ländern durchgeführt. Auch die Schweiz nahm an ausgewählten international vergleichenden Bildungsstudien teil, beispielsweise im Jahre 1995 an der Third International Mathematics and Science Study (TIMSS), seit 2000 in einem Drei-Jahres-Zyklus am Programme for International Student Assessment (PISA) sowie an weiteren, thematisch unterschiedlichen Erhebungen wie Adult Literacy and Life Skills (ALL), Civic Education Study (CIVED), International Civic and Citizenship Education Study (ICCS), International Computer and Information Literacy Study (ICILS) oder Teacher Education and Development Study in Mathematics (TEDS-M).

Viele Länder etablierten in diesem Zeitraum nationale Messungen der Bildungsstandards, um das Erreichen curricularer Bildungsziele zu überprüfen. Die Schweiz war im Jahr 2015 das einzige OECD-Mitgliedsland, das noch über keine Überprüfung der nationalen Bildungsziele verfügte (OECD, 2016). Es lässt sich demnach konstatieren, dass dem Schweizer Bildungsmonitoring die Erfassung curricularer Kompetenzen über alle Sprachregionen und Kantone hinweg lange Zeit fehlte. Zwar wurden die revidierten Bildungsartikel der Bundesverfassung bereits im Jahre 2006 durch das Schweizer Stimmvolk angenommen und rund ein Jahr später wurde mit der Interkantonalen Vereinbarung über die Harmonisierung der obligatorischen Schule festgelegt, dass das Erreichen der nationalen Bildungsziele in regelmässigen Abständen überprüft werden soll (Schweizerischen Konferenz der kantonalen Erziehungsdirektoren [EDK], 2007). Aber erst mit der Verabschiedung der Bildungsziele im Jahr 2011 und dem Formulieren von Grundkompetenzen zu bestimmten Zeitpunkten in der Schulkarriere und in verschiedenen Fächern (Schweizerische Konferenz der kantonalen Erziehungsdirektoren [EDK], 2011a, 2011b, 2011c, 2011d) wurde 
der Grundstein zur Überprüfung des Erreichens der Grundkompetenzen (ÜGK) in allen Kantonen der Schweiz gelegt.

Im Jahr 2016 erfolgte die erste Messung im Rahmen der ÜGK mit der Erhebung in Mathematik am Ende der obligatorischen Schulbildung (11. Schuljahr nach HarmoS ${ }^{1}$ ). Im Jahr 2017 wurde die ÜGK mit der Erhebung in der Schulsprache und der ersten Fremdsprache am Ende der Primarstufe (Schuljahr HarmoS 8) weitergeführt. Alle Schweizer Kantone beteiligten sich an der ÜGK, wobei schweizweit pro Erhebung mehr als $20^{\prime} 000$ Schülerinnen und Schüler getestet wurden. Im Unterschied zu anderen Schulleistungsstudien (z. B. PISA) liefert ÜGK keine Informationen über das gesamte Leistungsspektrum eines Fachbereichs, sondern fokussiert darauf, inwieweit die Schülerinnen und Schüler in den verschiedenen Kantonen und Sprachregionen die jeweiligen Grundkompetenzen, im Sinne von Minimalkompetenzen, erworben haben.

Neben den Leistungstests werden auf Ebene der Schülerinnen und Schüler mittels Kontextfragebogen zentrale Bedingungsfaktoren von schulischen Leistungen erfasst. Dabei werden fachliche und überfachliche individuelle Lernvoraussetzungen sowie verschiedene Aspekte der Lernumwelten der Schülerinnen und Schüler in den Blick genommen. Diese Daten liefern einerseits zusätzliche Informationen für das Bildungsmonitoring und erlauben andererseits die Untersuchung wissenschaftlicher Fragestellungen zur Erklärung von Unterschieden im Erreichen der Grundkompetenzen (Hasche,tet al., 2015; Hupka-Brunner et al., 2015).

Im Mai 2019 wurde der nationale Bericht der ÜGK 2016 (Konsortium ÜGK, 2019) zum Erreichen der mathematischen Grundkompetenzen im 11. Schuljahr HarmoS veröffentlicht. Die Ergebnisse zeigen, dass in der Schweiz 62 Prozent der Schülerinnen und Schüler die Grundkompetenzen in Mathematik erreichen. Dabei variieren die Anteile des Erreichens der Grundkompetenzen zwischen den Kantonen von 44 bis 83 Prozent. Analog zu anderen Studien spielen der Migrationsstatus, die zu Hause gesprochene Sprache und die soziale Herkunft eine Rolle, ob die Jugendlichen die mathematischen Grundkompetenzen erreichen. Die kantonalen Unterschiede lassen sich aufgrund der Schülerzusammensetzung in diesen Merkmalen jedoch kaum erklären. Ebenso lässt sich zwar nachweisen, dass in Kantonen mit mehr Unterrichtszeit für Mathematik ein etwas grösserer Schüleranteil die Grundkompetenzen erreichen. Aber auch diese kantonale Steuerungsgrösse erklärt nur einen geringen Teil der kantonalen Differenzen.

Die Ergebnisse zur ÜGK wurden in den Medien breit aufgenommen und lösten einen öffentlichen Diskurs zur Harmonisierung des Bildungswesens aus. $\mathrm{Zu}$ reden gab der Befund, dass mehr als ein Drittel der Jugendlichen die vorgegebenen Bildungsziele in Mathematik nicht erreichte. Ausserdem war die Frage nach möglichen Gründen, weshalb in einigen Kantonen höhere Schüleranteile die grundlegenden Bildungsziele erreichen als in anderen, von grossem Interesse.

Zur Beschreibung des komplexen Bedingungsgefüges von schulischen Leistungen hat sich in der Bildungsforschung mittlerweile die Denkfigur des Angebots-Nutzungs-Modells weitgehend durchgesetzt (Brühwiler et al., 2017). 
Demnach ist der Unterricht als ein Angebot aufzufassen, das von den Lernenden in angemessener Weise genutzt werden kann. Erst über diese spezifischen Lernaktivitäten erfolgt die individuelle Wissenskonstruktion. Der Lernprozess hängt dabei nicht nur vom Unterricht $a b$, sondern auch von den individuellen Lernvoraussetzungen sowie Kontextfaktoren wie die familiäre Herkunft, die Zusammensetzung der Schulklasse, schulische oder auch gesellschaftliche Rahmenbedingungen (Seidel, 2014). Dementsprechend lassen sich die wesentlichen Bedingungsfaktoren von schulischen Leistungen grob in die folgenden drei Ebenen unterscheiden (Helmke, 2017):

- Kontextfaktoren bzw. Aspekte der Lernumwelten (z. B. gesellschaftliche Rahmenbedingungen, Schulumwelt, Klassenkontext, Erziehungsverhalten und Erwartungen der Eltern),

- Merkmale des Unterrichts (z. B. Lehr- und Lernmethoden, Aufgabenqualität, Lernstrategien, kognitive Aktivierung) sowie

- individuelle Lernvoraussetzungen (z. B. Vorwissen, Motivation, Interesse, Emotion, Selbstkonzept, Einstellungen).

In diesem Rahmenmodell werden schulische Leistungen als das Ergebnis einer Wirkungskette verstanden, das sowohl distale als auch proximale Faktoren umfasst (Helmke \& Weinert, 1997). Während distale Faktoren (z.B. gesellschaftliche Rahmenbedingungen oder das Bildungssystem) weit vom Zielkriterium Schulleistung entfernt sind und nur indirekt auf den Lernerfolg wirken, sind proximale Faktoren (z.B. kognitive oder motivationale Lernvoraussetzungen), die unmittelbar auf den Lernprozess einwirken, für die Erklärung von Schulleistungen viel bedeutsamer (Brühwiler et al., 2017).

Das vorliegende Themenheft vertieft die bereits erfolgte nationale Berichterstattung, welche in erster Linie soziodemografische Merkmale sowie einzelne systemische Faktoren als Erklärungsgrössen für Unterschiede im Erreichen der Grundkompetenzen heranzog. In den sechs Beiträgen wird der übergeordneten Frage nachgegangen, welche Faktoren auf der Ebene des Kontexts, des Unterrichts oder auf der individuellen Ebene mit dem Erreichen der Grundkompetenzen in einem Zusammenhang stehen. Jeder Artikel fokussiert jeweils einen oder mehrere Bedingungsfaktoren schulischer Leistungen, welche sich in diesen drei Ebenen verorten lassen.

Die ersten beiden Beiträge thematisieren die Bedeutung von Kontextmerkmalen auf der Ebene der Schule für das Erreichen von Grundkompetenzen. Franck Petrucci, Alice Ambrosetti, Francesca Crotta und Christian Nidegger untersuchten in den Kantonen Tessin und Genf, inwiefern die Zusammensetzung der Schülerschaft an Schulen nach Merkmalen der sozialen Herkunft und der schulischen Leistung mit dem Erreichen der Grundkompetenzen zusammenhängen. Die Ergebnisse zeigen in beiden Kantonen nur moderate Effekte aufgrund der Schülercharakteristika. Dies führt zur Diskussion, welche Informationen zum Schulkontext im Rahmen einer Bildungsmonitoringstudie gesammelt werden 
müssten, um kantonale Unterschiede in den Leistungen besser erklären zu können.

Im zweiten Artikel vergleichen Francesca Crotta, Miriam Salvisberg und Giovanna Zanolla das Erreichen der mathematischen Grundkompetenzen der jeweiligen Schülerin bzw. des jeweiligen Schülers mit der durch die Lehrperson vergebenen Schulnote in Mathematik. Die Analysen zeigen, dass im Schultyp mit grundlegenden Anforderungen das Erreichen einer (mindestens) genügenden Note nicht gewährleistet, dass die Schülerinnen und Schüler die im Rahmen von ÜGK gemessenen Grundkompetenzen in Mathematik erlangen. Auch bei gleichen Noten und innerhalb desselben Anforderungsniveaus spielt es für die Bildungsentscheide der Jugendlichen eine Rolle, ob sie die Grundkompetenzen in Mathematik erreichen oder nicht.

Merkmale auf der Ebene des Unterrichts werden von Andrea B. Erzinger, Boris Eckstein und Christian Brühwiler in den Fokus genommen, die nach unterrichtlichen Gelingensbedingungen für das Erreichen mathematischer Grundkompetenzen fragen. Logistische Regressionsanalysen ergeben, dass ein störungsarmer, von hoher Instruktionsklarheit und Autonomieunterstützung geprägter Unterricht mit einer erhöhten Wahrscheinlichkeit einhergeht, die mathematischen Grundkompetenzen zu erreichen. Die Bedeutung der Unterrichtsmerkmale zeigen sich abhängig vom besuchten schulischen Anforderungsniveau: Die Wahrscheinlichkeit, die Grundkompetenzen zu erreichen erhöht sich in den untersuchten Schultypen je nach Einschätzung der Unterrichtsqualität durch die Schülerinnen und Schüler differenziell.

Die drei abschliessenden Beiträge des Thementeils fokussieren auf individuelle Lernvoraussetzungen zur Erklärung von Unterschieden im Erreichen der Grundkompetenzen in Mathematik. Tina Hascher und Gerda Hagenauer untersuchten das Wohlbefinden von Schülerinnen und Schülern in der Schweiz. Sie stellen fest, dass Jugendliche über positive Einstellungen und ein gutes akademisches Selbstkonzept verfügen sowie wenig physische Beschwerden und soziale Probleme aufweisen. Es lässt sich jedoch ein Mangel an Freude und eine Prävalenz für Sorgen in der Schule feststellen. Weiter zeigen sich Unterschiede hinsichtlich Geschlecht, Sprachregion, Migrationshintergrund und Schultyp sowie Zusammenhänge zwischen Wohlbefinden und Schulverdrossenheit bzw. Schulschwänzen. Die Ergebnisse bekräftigen die Bedeutsamkeit des Wohlbefindens von Jugendlichen als ein zentrales Kriterium für Schulqualität.

Ben Jann und Sandra Hupka gehen in ihrem Beitrag der geschlechtsspezifischen Diskrepanz zwischen mathematischen Kompetenzen und der Selbsteinschätzung der Schülerinnen und Schüler in Bezug auf die Wahl eines MINT2Berufes nach. Sie weisen nach, dass männliche Jugendliche überdurchschnittlich häufiger erwarten, mit 30 Jahren einen Beruf als MINT-Fachkraft auszuüben. Weiter zeigen ihre Analysen, dass Frauen ihre mathematischen Kompetenzen häufiger unterschätzten als Männer. Zudem hängen die beruflichen Aspirationen im MINT-Bereich sowohl vom mathematischen Selbstkonzept als auch von der 
mathematischen Selbstwirksamkeit ab. Junge Frauen sehen - unabhängig von den entsprechenden mathematischen Kompetenzen - ihre berufliche Zukunft seltener im MINT-Bereich als junge Männer. Die Ergebnisse werden vor dem Hintergrund der Untervertretung von Frauen in MINT-Berufen und des Fachkräftemangels im MINT-Bereich diskutiert.

Schliesslich thematisieren Boris Girnat, Gerda Hagenauer und Tina Hascher auf der Basis der Erwartungs-mal-Wert-Theorie den Zusammenhang von Schülermotivation und Leistungen in der Mathematik. Die Ergebnisse bestätigen den hohen prädiktiven Wert von Lernzielorientierungen, Selbstkonzept und Selbstwirksamkeitserwartung für die Mathematikleistung. Geschlechtsspezifische Unterschiede, etwa das deutlich geringere mathematische Selbstkonzept von Mädchen gegenüber Knaben trotz vergleichbarer Leistungen, lassen sich durch eine mehrdimensionale Erfassung der mathematischen Selbstwirksamkeitserwartung differenzieren. So lassen sich signifikante Geschlechterunterschiede in der angewandten Mathematik, nicht jedoch in der Algebra feststellen. Bezüglich der Zielorientierungen bestehen ebenfalls Geschlechterunterschiede: Knaben weisen in allen Facetten höhere Werte auf als Mädchen. Allerdings sind die Effekte geringer als bei den meisten Skalen der Selbstwirksamkeitserwartung.

\section{Anmerkungen}

1 Mit dem 11. Schuljahr wird auf die HarmoS-Zählweise referenziert, bei der die ersten beiden Schuljahre den beiden Jahren im Kindergarten entsprechen. Es handelt sich demnach beim 11. Schuljahr um die 9. Klassenstufe.

2 MINT steht als Akronym für 'Mathematik, Informatik, Naturwissenschaften und Technik'.

\section{Literaturverzeichnis}

Brühwiler, C., Helmke, A. \& Schrader, F.-W. (2017). Determinanten der Schulleistung. In M. K. W. Schweer (Hrsg.), Lehrer-Schüler-Interaktion. Inhaltsfelder, Forschungsperspektiven und methodische Zugänge (3., S. 291-314). Springer VS. https://doi.org/10.1007/978-3531-91104-5

Hascher, T., Brühwiler, C., Erzinger, A. \& Girnat, B. G. H. (2015). Erläuterungen zu den Skalen "Mathematik lernen" und "Grundeinstellungen zur Mathematik». Unveröffentlichter Bericht: Universität Bern, Pädagogische Hochschule St.Gallen, Pädagogische Hochschule Fachhochschule Nordwestschweiz.

Helmke, A. (2017). Unterrichtsqualität und Lehrerprofessionalität. Diagnose, Evaluation und Verbesserung des Unterrichts (7. überarb. Auflage). Klett-Kallmeyer.

Helmke, A. \& Weinert, F. E. (1997). Bedingungsfaktoren schulischer Leistungen. In F. E. Weinert (Hrsg.), Psychologie des Unterrichts und der Schule (S. 71-176). Hogrefe.

Hupka-Brunner, S., Jann, B., Meyer, T., Imdorf, C., Sacchi, S., Müller, B. et al. (2015). Erläuterungen zum Kontextfragebogen der ÜGK 2016: Allgemeiner Teil. Unveröffentlichter Bericht: Universität Bern.

Konsortium ÜGK (Hrsg.). (2019). Überprïfung der Grundkompetenzen. Nationaler Bericht der ÜGK 2016: Mathematik 11. Schuljahr. https://doi.org/10.18747/PHSG-coll3/id/386

OECD (2016). PISA 2015 Results (Volume II). Policies and practices for successful schools. OECD. https://doi.org/10.1787/9789264267510-en 
Ryan, K. E. \& Shepard, L. A. (2008). The future of test-based educational accountability. Routledge.

Schweizerische Konferenz der kantonalen Erziehungsdirektoren (2011a). Grundkompetenzen für die Fremdsprachen. Nationale Bildungsstandards. EDK. Zugriff am 29.06.2020. Verfügbar unter https://edudoc.ch/record/96780/files/grundkomp_fremdsprachen_d.pdf

Schweizerische Konferenz der kantonalen Erziehungsdirektoren (2011b). Grundkompetenzen für die Mathematik. Nationale Bildungsstandards. EDK. Zugriff am 29.06.2020. Verfügbar unter https://edudoc.ch/record/96784/files/grundkomp_math_d.pdf

Schweizerische Konferenz der kantonalen Erziehungsdirektoren (2011c). Grundkompetenzen für die Naturwissenschaften. Nationale Bildungsstandards. EDK. Zugriff am 29.06.2020. Verfügbar unter https://edudoc.ch/record/96787/files/grundkomp_nawi_d.pdf

Schweizerische Konferenz der kantonalen Erziehungsdirektoren (2011d). Grundkompetenzen für die Schulsprache. Nationale Bildungsstandards. EDK. Zugriff am 29.06.2020. Verfügbar unter https://edudoc.ch/record/96791/files/grundkomp_schulsprache_d.pdf

Schweizerischen Konferenz der kantonalen Erziehungsdirektoren (2007). Interkantonale Vereinbarung über die Harmonisierung der obligatorischen Schule (HarmoS-Konkordat). Zugriff am 07.07.2020. Verfügbar unter https://edudoc.ch/record/24711/files/ HarmoS_d.pdf

Seidel, T. (2014). Angebots-Nutzungs-Modelle in der Unterrichtspsychologie. Integration von Struktur- und Prozessparadigma. Zeitschrift für Pädagogik, 60(6), 850-866. 